\title{
Specialty Grand Challenge for Brain Disease Mechanisms
}

\author{
Detlev Boison* \\ Department of Neurosurgery, Robert Wood Johnson Medical School, Rutgers University, Piscataway, NJ, United States
}

Keywords: disease burden, disease mechanism, disease modification, network pharmacology, epigenetics, global approaches, neurological disorders, CNS disorders

\section{GLOBAL BURDEN OF CNS DISORDERS}

The global burden of CNS diseases is expected to increase dramatically within the next decades. A recent position paper in the Annals of Neurology estimates the annual cost of neurological diseases affecting nearly 100 million Americans to be in the range of 800 billion dollars [including $\$ 37 \mathrm{~B}$ for epilepsy, $\$ 86 \mathrm{~B}$ for traumatic brain injury (TBI), and $\$ 110 \mathrm{~B}$ for stroke] and calls for the development of preventative and disease-modifying therapies (Gooch et al., 2017). Indeed, conventional therapies are largely symptomatic and do not influence the genesis or progression of the disease. A major gap and challenge for the development of novel disease modifying therapies is the transition from target-centric (symptomatic) approaches to globally-acting multi-modal approaches, which are able to restore complex network function in the brain. It is therefore time to develop innovative new concepts to understand better the interconnectedness of the CNS and to translate new knowledge into novel therapeutics. To achieve this goal Big Data approaches will play a major role in connecting findings from a multitude of different mechanisms and disciplines. The multidisciplinary and integrative scope of Brain Disease Mechanisms will help to bring different ideas from different disciplines under one common roof.

Edited and reviewed by: Jochen C. Meier,

Technische Universitat Braunschweig, Germany

*Correspondence: Detlev Boison detlev.boison@rutgers.edu

Specialty section:

This article was submitted to Brain Disease Mechanisms,

a section of the journal Frontiers in Molecular Neuroscience

Received: 01 April 2021 Accepted: 13 April 2021 Published: 10 May 2021

Citation:

Boison D (2021) Specialty Grand

Challenge for Brain Disease

Mechanisms.

Front. Mol. Neurosci. 14:689903. doi: 10.3389/fnmol.2021.689903

\section{NEW CHALLENGES AS A CONSEQUENCE OF COVID-19?}

The outbreak of the novel coronavirus disease 2019 (COVID-19) caused by Severe Acute Respiratory Syndrome CoronaVirus-2 (SARS-CoV-2) may lead to new challenges and to a further increase in the global burden of CNS diseases. It has now become clear that SARS-CoV-2 can also attack the nervous system (Zubair et al., 2020), and it is estimated that at least 40\% of COVID-19 patients develop neurological complications (Liotta et al., 2020), including encephalitis, increased risk of stroke, and injuries due to lack of oxygen (Fridman et al., 2020; Kantonen et al., 2020; Paterson et al., 2020). A wide range of injuries to the brain in turn, including stroke, traumatic brain injury, and brain infection, are known to constitute a primary cause for the development of acquired epilepsies including temporal lobe epilepsy (Klein et al., 2018). Because the latent period for the development of acquired epilepsies in humans is in the range of months to years it is still too early to assess whether COVID-19 might be linked to a future increase in epilepsy cases.

An additional unknown are the consequences of SARS-CoV-2 infections during pregnancy. A leading hypothesis for the etiology of neurodevelopmental disorders such as autism or schizophrenia suggests that maternal immune activation caused by viral infections during pregnancy might play a major causative role for the derailment of developmental processes critical for normal brain development (Canetta and Brown, 2012; Lombardo et al., 2018). Again, it is 
still too early to assess whether SARS-CoV-2 infections during pregnancy can be linked to the development of autism or schizophrenia.

The examples shown above illustrate that brain diseases and associated etiologies and mechanisms are a constantly evolving field, which may need concerted efforts to accelerate new research directions.

\section{NEW FRONTIERS IN DISEASE MECHANISMS}

Why should we be interested in disease mechanisms? The ultimate goal, obviously, is that better understanding of disease mechanisms leads to better treatment options for persons affected by brain disorders and to the reduction of the global health burden. Four areas deserve increased attention during the next decade:

\section{Disease Modification}

The biggest challenge for the reduction of the global health burden of neurological conditions is a paradigm shift from symptomatic to disease modifying treatments. Up until now, pharmacological treatment options are largely symptomatic. For example, antiseizure drugs (ASDs) have been designed to reduce neuronal excitability, and thereby seizures, the dominant symptom of epilepsy. They do so mostly by affecting ion channels and neurotransmitter release (Sills and Rogawski, 2020). However, despite the development of about 30 new ASDs over the past 30 years, treatment outcomes for persons with epilepsy have not significantly been improved and about one third of all persons with epilepsy remain refractory to pharmacological treatment (Chen et al., 2018). Most currently used ASDs have been discovered in screens designed to detect seizure suppression in rodent seizure models. As a default of this screening approach, compounds are identified, which have the capability to suppress the dominant symptom of epilepsy, which is the seizure. As becomes obvious, seizurebased drug screens are not able to identify compounds, which can treat comorbidities of epilepsy, such as depression, anxiety, or cognitive impairment, or which affect disease progression and epilepsy development. Therefore, there is a major unmet need to identify treatment options, which are disease modifying and thereby affect fundamental mechanisms implicated in pathogenic processes leading to disease and its progression. Treatments, which prevent disease or its progression would be a game changer not only for epilepsy but also for progressive neurodegenerative diseases such as Alzheimer's or Parkinson's disease. In order to develop novel disease modifying treatments, a better understanding of the interconnectedness of the CNS on a multi-omics level becomes a necessity.

\section{Network Approaches}

As opposed to the design of highly selective ligands that bind on individual targets, network pharmacology holds the promise to affect several beneficial targets simultaneously, an approach suitable to increase efficacy and reduce toxicity (Hopkins, 2008). Those network based approaches can be based on a rational design. If several mechanisms are involved in disease development and progression, it can be assumed that those mechanisms are connected by multiple nodes. Identification of central nodes and targeting them in a rationally designed network approach holds the promise to reconstruct network function implicated in disease pathogenesis. A proof of principle for the utility of this approach has been demonstrated recently by demonstrating that a combination of levetiracetam and topiramate altered multiple epileptogenesisrelevant targets and provided robust disease modifying effects (Schidlitzki et al., 2020). A promising alternative to targetcentric conventional pharmacology is the development of biochemical interventions for the treatment of disease. It is now well-established that biochemical alterations are implicated in the pathophysiology of a majority of neurological conditions (Boison, 2016). Specifically, core metabolites, such as adenosine are uniquely linked to energy homeostasis (ATP), used as building blocks of biomolecules (RNA, including poly-A tails of mRNAs), coupled to transmethylation reactions (DNA and histone methylation), and act as receptor ligands (adenosine receptors) (Boison, 2013; Boison and Yegutkin, 2019). Thus, adenosine is a unique network regulator linking metabolism and gene expression with neuromodulation. Strikingly, adenosine deficiency is a common pathological hallmark of epilepsy, Alzheimer's disease, Parkinson's disease, amyotrophic lateral sclerosis, and can be the direct explanation for a wide range of symptoms including seizures, sleep alterations, depression, and changes in cognition and affective behavior (Li et al., 2008; Shen et al., 2012; Hines et al., 2013; Boison and Aronica, 2015). Consequently, adenosine augmentation therapies, e.g., through engineered stem cells (Fedele et al., 2004; Li et al., 2009), are uniquely suited to restore network homeostasis, and to not only suppress comorbid symptoms but also to exert lasting disease modifying therapeutic effects ( $\mathrm{Li}$ et al., 2008; Boison, 2012; Shen et al., 2012; Williams-Karnesky et al., 2013). The concept of network approaches is not new. This year marks the 100th anniversary of the high-fat, lowcarbohydrate ketogenic diet, a metabolic therapy, which has successfully been used for the treatment of epilepsy for decades (Neal et al., 2008; Kossoff, 2010; Stafstrom and Rho, 2012). Recent studies suggest strongly that metabolic approaches may have underappreciated antiepileptogenic and disease-modifying properties (Muller-Schwarze et al., 1999; Todorova et al., 2000; Lusardi et al., 2015; Boison, 2017; Boison and Rho, 2020) and provide benefits to a wide spectrum of additional conditions including pain, autism, brain cancer, and Alzheimer's disease (Ruskin et al., 2009, 2017; Brownlow et al., 2013; Chung and Park YRationale, 2017; Vergati et al., 2017). The benefits of metabolic therapies can be explained by combining several different mechanisms which affect network homeostasis on the levels of energy equilibrium, mitochondrial function, changes in metabolites and neurotransmitters, and epigenetic reprogramming (Kobow et al., 2013; Rogawski et al., 2016; Boison, 2017; Augustin et al., 2018; Boison and Rho, 2020). 
The examples above demonstrate the promise of network based treatment approaches and suggest that the biochemistry of brain disease is a new frontier to understand the complexity and interconnectedness involved in etiopathological processes. Fully understanding those mechanisms will provide the basis for rationally designed multimodal therapeutics uniquely suited to exert disease modifying properties and to treat complex comorbid syndromes.

\section{Epigenetics}

We are living in the post genomic age. Genetic mutations and polymorphisms yield cues for our understanding of CNS disorders and the development of personalized medicines. A recent PubMed search for "genomic" yielded 1.6 million hits. In contrast, the term "epigenomic" yielded only 18,000 hits. This discrepancy is surprising, given the fact that epigenetic alterations, which include modifiable changes in DNA methylation, histone methylation and acetylation, and the expression of non-coding RNAs, regulate the expression of the very genes that are the focus of genomics-based research efforts. Because epigenetic mechanisms regulate gene expression, there is an urgent need to invest in the field of epigenomics which is one of the remaining frontiers in neuroscience. The study of epigenetics and the development of "epigenetic medicines" is relatively well-developed in the field of cancer (Du et al., 2015; Huang et al., 2015; Zahnow et al., 2016), however in its infancy in our understanding of neurological disorders. For the rigorous assessment of epigenomic data sets it will be important to standardize data and methods as even minor protocol variations can have major impact on epigenomic data sets. Epigenetic changes are subject to metabolic regulation (Kobow et al., 2013; WilliamsKarnesky et al., 2013; Boison and Rho, 2020; Kuchukulla and Boison, 2020) and thus may provide an interface between environment, metabolism, and gene expression. Of crucial importance for the understanding of disease mechanisms is the interconnectedness of physiological, molecular, cellular, metabolic, epigenetic, and genetic mechanisms at the subcellular, cellular, and regional levels.

\section{Global Approaches}

Finally, the burden of CNS diseases is global with tremendous regional disparities. It is not enough to focus on Alzheimer's disease, which is a prevalent problem specifically in wealthy countries with a high and rising life expectancy. Strikingly, according to data from the World Health Organization, Alzheimer's disease is five times more prevalent in high income countries as compared to low income countries, whereas meningitis is 13 times more prevalent in low income countries as compared to high income countries. Other, neglected conditions, which affect a significant share of the world population require equal attention. For example, cerebral malaria, the most severe neurological manifestation of severe malaria, has an incidence of $1,120 / 100,000 /$ year in endemic areas of Africa. It is estimated that a minimum of 575,000 children in Africa develop cerebral malaria annually (Breman, 2001; Murphy and Breman, 2001). Consequences of cerebral malaria include long-term cognitive impairment (25\%), speech and language impairment (11.8\%), epilepsy (10\%), as well as behavioral and neuropsychiatric disorders (Idro et al., 2010a,b). Those examples show major disparities in the distribution of the global burden of neurological diseases; therefore it becomes a moral necessity to invest more research into global health issues. Related to this, there is a risk of introducing bias in clinical trial design. It is important to design trials appropriately to make sure that resulting treatments work equally well across diverse population groups.

\section{THE NEED FOR PUBLIC ENGAGEMENT}

The current COVID crisis has shown that public distrust in science can have catastrophic impacts on case numbers. The combination of distrust in science and medical populism has led to avoidable surges in COVID infections and deaths, specifically in countries where leaders have discredited scientific knowledge and findings, by downplaying the impacts of the pandemic, by promoting easy and scientifically unfounded solutions or treatments, and by forging divisions between believers and nonbelievers of science (Lasco, 2020; Hotez, 2021). On the other hand, science has produced a remarkable success story: the development of new vaccines to a new virus within a record breaking time frame of $<1$ year. There is hope that, if the vaccines work in significantly reducing the impacts of the pandemic and allow the return to a new normal, there will be a boost for the general trust in science. Currently, about three quarters of the population agree that science and technology make our lives better and that scientists contribute to major medical advances. This implies that scientists have a responsibility to maintain and expand this level of trust by serving the public. It means that the fruits of scientific discovery need to be shared broadly with our communities and that scientists need to build trust by demonstrating that science is not done in silos, but that scientists are members of the public and that the public is part of the scientific community. Engaging the public will be key in fighting the pandemic and there is hope that advances in fighting the pandemic will instill trust in the scientific process.

\section{CONCLUSIONS}

Frontiers in Molecular Neuroscience is a leading journal in the area of Neuroscience publishing rigorously peer-reviewed articles with a focus on molecular mechanisms implicated in health and disease of the nervous system. Its Brain Disease Mechanisms section focuses on key pathways and molecular mechanisms involved in the genesis, progression, and maintenance of central nervous system pathologies including neurodevelopmental, neurodegenerative, neuroinflammatory, and neuropsychiatric diseases, including insults such as stroke, traumatic and spinal cord injuries, and infection, as well as mechanisms linking injury to downstream consequences such as epilepsy or neuropathic pain. Of crucial importance for the understanding of disease mechanisms is the interconnectedness 
of physiological, molecular, cellular, metabolic, epigenetic, and genetic mechanisms at the subcellular, cellular, and regional levels. A specific interest is the exploration of disease mechanisms and their translation into novel targeted therapeutic approaches. Brain Disease Mechanisms thereby provides an interdisciplinary platform for new developments in this highly complex field that demands the involvement of a broad range of professionals and the public to create a forum for the exchange of knowledge and the global dissemination of science.

\section{REFERENCES}

Augustin, K., Khabbush, A., Williams, S., Eaton, S., Orford, M., Cross, J. H., et al. (2018). Mechanisms of action for the medium-chain triglyceride ketogenic diet in neurological and metabolic disorders. Lancet Neurol. 17, 84-93. doi: 10.1016/S1474-4422(17)30408-8

Boison, D. (2012). “Adenosine augmentation therapy," in Jasper's Basic Mechanisms of the Epilepsies, eds J. L. Noebels, M. Avoli, M. A. Rogawski, R. W. Olsen, and A. V. Delgado-Escueta (Bethesda, MD: Oxford University Press), 1150-1160.

Boison, D. (2013). Adenosine kinase: exploitation for therapeutic gain. Pharmacol. Rev. 65, 906-943. doi: 10.1124/pr.112.006361

Boison, D. (2016). The biochemistry and epigenetics of epilepsy: focus on adenosine and glycine. Front. Mol. Neurosci. 9:26. doi: $10.3389 /$ fnmol.2016.00026

Boison, D. (2017). New insights into the mechanisms of the ketogenic diet. Curr. Opin. Neurol. 30, 187-192. doi: 10.1097/WCO.0000000000000432

Boison, D., and Aronica, E. (2015). Comorbidities in neurology: is adenosine the common link? Neuropharmacology 97, 18-34. doi: 10.1016/j.neuropharm.2015.04.031

Boison, D., and Rho, J. M. (2020). Epigenetics and epilepsy prevention: the therapeutic potential of adenosine and metabolic therapies. Neuropharmacology 167:107741. doi: 10.1016/j.neuropharm.2019.107741

Boison, D., and Yegutkin, G. G. (2019). Adenosine metabolism: emerging concepts for cancer therapy. Cancer Cell 36, 582-596. doi: 10.1016/j.ccell.2019.10.007

Breman, J. G. (2001). The ears of the hippopotamus: manifestations, determinants, and estimates of the malaria burden. Am. J. Trop. Med. Hyg. 64, 1-11. doi: 10.4269/ajtmh.2001.64.1

Brownlow, M. L., Benner, L., D’Agostino, D., Gordon, M. N., and Morgan, D. (2013). Ketogenic diet improves motor performance but not cognition in two mouse models of Alzheimer's pathology. PLoS ONE 8:e75713. doi: 10.1371/journal.pone.0075713

Canetta, S. E., and Brown, A. S. (2012). Prenatal infection, maternal immune activation, and risk for schizophrenia. Transl. Neurosci. 3, 320-327. doi: 10.2478/s13380-012-0045-6

Chen, Z., Brodie, M. J., Liew, D., and Kwan, P. (2018). Treatment outcomes in patients with newly diagnosed epilepsy treated with established and new antiepileptic drugs: a 30-year longitudinal cohort study. JAMA Neurol. 75, 279-286. doi: 10.1001/jamaneurol.2017.3949

Chung, H. Y., and Park YRationale, K. (2017). Feasibility and acceptability of ketogenic diet for cancer treatment. J. Cancer Prev. 22, 127-134. doi: 10.15430/JCP.2017.22.3.127

Du, J., Johnson, L. M., Jacobsen, S. E., and Patel, D. J. (2015). DNA methylation pathways and their crosstalk with histone methylation. Nat. Rev. Mol. Cell Biol. 16, 519-532. doi: 10.1038/nrm4043

Fedele, D. E., Koch, P., Brüstle, O., Scheurer, L., Simpson, E. M., Mohler, H., et al. (2004). Engineering embryonic stem cell derived glia for adenosine delivery. Neurosci. Lett. 370, 160-165. doi: 10.1016/j.neulet.2004.08.031

Fridman, S., Bres Bullrich, M., Jimenez-Ruiz, A., Costantini, P., Shah, P., Just, C., et al. (2020). Stroke risk, phenotypes, and death in COVID-19: systematic review and newly reported cases. Neurology 95, e3373-e3385. doi: 10.1212/WNL.0000000000010851

Gooch, C. L., Pracht, E., and Borenstein, A. R. (2017). The burden of neurological disease in the United States: a summary report and call to action. Ann. Neurol. 81, 479-484. doi: 10.1002/ana.24897

\section{AUTHOR CONTRIBUTIONS}

DB designed and wrote the manuscript.

\section{FUNDING}

DB was supported through grants from the National Institutes of Health (NIH, R01-NS103740, R01-NS065957) and CURE Epilepsy.

Hines, D. J., Schmitt, L. I., Hines, R. M., Moss, S. J., and Haydon, P. G. (2013). Antidepressant effects of sleep deprivation require astrocytedependent adenosine mediated signaling. Transl. Psychiatry 3:e212. doi: $10.1038 /$ tp. 2012.136

Hopkins, A. L. (2008). Network pharmacology: the next paradigm in drug discovery. Nat. Chem. Biol. 4, 682-690. doi: 10.1038/nchembio.118

Hotez, P. J. (2021). Anti-science kills: from Soviet embrace of pseudoscience to accelerated attacks on US biomedicine. PLoS Biol. 19:e3001068. doi: 10.1371/journal.pbio.3001068

Huang, W. Y., Hsu, S. D., Huang, H. Y., Sun, Y. M., Chou, C. H., Weng, S. L., et al. (2015). MethHC: a database of DNA methylation and gene expression in human cancer. Nucleic Acids Res. 43, D856-D861. doi: 10.1093/nar/gku1151

Idro, R., Kakooza-Mwesige, A., Balyejjussa, S., Mirembe, G., Mugasha, C., Tugumisirize, J., et al. (2010b). Severe neurological sequelae and behaviour problems after cerebral malaria in Ugandan children. BMC Res. Notes 3:104. doi: 10.1186/1756-0500-3-104

Idro, R., Marsh, K., John, C. C., and Newton, C. R. (2010a). Cerebral malaria: mechanisms of brain injury and strategies for improved neurocognitive outcome. Pediatr. Res. 68, 267-274. doi: 10.1203/PDR.0b013e3181eee738

Kantonen, J., Mahzabin, S., Mayranpaa, M. I., Tynninen, O., Paetau, A., Andersson, N., et al. (2020). Neuropathologic features of four autopsied COVID-19 patients. Brain Pathol. 30, 1012-1016. doi: 10.1111/bpa.12889

Klein, P., Dingledine, R., Aronica, E., Bernard, C., Blumcke, I., Boison, D., et al. (2018). Commonalities in epileptogenic processes from different acute brain insults: do they translate? Epilepsia 59, 37-66. doi: 10.1111/epi.13965

Kobow, K., Kaspi, A., Harikrishnan, K. N., Kiese, K., Ziemann, M., Khurana, I., et al. (2013). Deep sequencing reveals increased DNA methylation in chronic rat epilepsy. Acta Neuropathol. 126, 741-756. doi: 10.1007/s00401-013-1168-8

Kossoff, E. H. (2010). The ketogenic diet: an appropriate first-line therapy? Expert Rev. Neurother. 10, 843-845. doi: 10.1586/ern.10.35

Kuchukulla, M., and Boison, D. (2020). Are glia targets for neuropathic orofacial pain therapy? J. Am. Dent. Assoc. S0002-8177(20)30377-9. doi: 10.1016/j.adaj.2020.05.017. [Epub ahead of print].

Lasco, G. (2020). Medical populism and the COVID-19 pandemic. Glob. Public Health 15, 1417-1429. doi: 10.1080/17441692.2020.1807581

Li, T., Ren, G., Kaplan, D. L., and Boison, D. (2009). Human mesenchymal stem cell grafts engineered to release adenosine reduce chronic seizures in a mouse model of CA3-selective epileptogenesis. Epilepsy Res. 84, 238-241. doi: 10.1016/j.eplepsyres.2009.01.002

Li, T., Ren, G., Lusardi, T., Wilz, A., Lan, J. Q., Iwasato, T., et al. (2008). Adenosine kinase is a target for the prediction and prevention of epileptogenesis in mice. J. Clin. Invest. 118, 571-582. doi: 10.1172/JCI33737

Liotta, E. M., Batra, A., Clark, J. R., Shlobin, N. A., Hoffman, S. C., Orban, Z. S., et al. (2020). Frequent neurologic manifestations and encephalopathyassociated morbidity in Covid-19 patients. Ann. Clin. Transl. Neurol. 7, 2221-2230. doi: 10.1002/acn3.51210

Lombardo, M. V., Moon, H. M., Su, J., Palmer, T. D., Courchesne, E., and Pramparo, T. (2018). Maternal immune activation dysregulation of the fetal brain transcriptome and relevance to the pathophysiology of autism spectrum disorder. Mol. Psychiatry 23, 1001-1013. doi: 10.1038/mp.2017.15

Lusardi, T. A., Akula, K. K., Coffman, S. Q., Ruskin, D. N., Masino, S. A., and Boison, D. (2015). Ketogenic diet prevents epileptogenesis and disease progression in adult mice and rats. Neuropharmacology 99, 500-509. doi: 10.1016/j.neuropharm.2015.08.007 
Muller-Schwarze, A. B., Tandon, P., Liu, Z., Yang, Y., Holmes, G. L., and Stafstrom, C. E. (1999). Ketogenic diet reduces spontaneous seizures and mossy fiber sprouting in the kainic acid model. Neuroreport 10, 1517-1522. doi: 10.1097/00001756-199905140-00023

Murphy, S. C., and Breman, J. G. (2001). Gaps in the childhood malaria burden in Africa: cerebral malaria, neurological sequelae, anemia, respiratory distress, hypoglycemia, and complications of pregnancy. Am. J. Trop. Med. Hyg. 64, 57-67. doi: 10.4269/ajtmh.2001.64.57

Neal, E. G., Chaffe, H., Schwartz, R. H., Lawson, M. S., Edwards, N., Fitzsimmons, G., et al. (2008). The ketogenic diet for the treatment of childhood epilepsy: a randomised controlled trial. Lancet Neurol. 7, 500-506. doi: 10.1016/S1474-4422(08)70092-9

Paterson, R. W., Brown, R. L., Benjamin, L., Nortley, R., Wiethoff, S., Bharucha, T., et al. (2020). The emerging spectrum of COVID-19 neurology: clinical, radiological, and laboratory findings. Brain 143, 3104-3120. doi: 10.1093/brain/awaa240

Rogawski, M. A., Loscher, W., and Rho, J. M. (2016). Mechanisms of action of antiseizure drugs and the ketogenic diet. Cold Spring Harb. Perspect. Med. 6:a022780. doi: 10.1101/cshperspect.a022780

Ruskin, D. N., Fortin, J. A., Bisnauth, S. N., and Masino, S. A. (2017). Ketogenic diets improve behaviors associated with autism spectrum disorder in a sex-specific manner in the EL mouse. Physiol. Behav. 168, 138-145. doi: 10.1016/j.physbeh.2016.10.023

Ruskin, D. N., Kawamura, M., and Masino, S. A. (2009). Reduced pain and inflammation in juvenile and adult rats fed a ketogenic diet. PLOS ONE 4:e8349. doi: 10.1371/journal.pone.0008349

Schidlitzki, A., Bascunana, P., Srivastava, P. K., Welzel, L., Twele, F., Tollner, K., et al. (2020). Proof-of-concept that network pharmacology is effective to modify development of acquired temporal lobe epilepsy. Neurobiol. Dis. 134:104664. doi: 10.1016/j.nbd.2019.104664

Shen, H. Y., Singer, P., Lytle, N., Wei, C. J., Lan, J. Q., Williams-Karnesky, R. L., et al. (2012). Adenosine augmentation ameliorates psychotic and cognitive endophenotypes of schizophrenia. J. Clin. Invest. 122, 2567-2577. doi: 10.1172/JCI62378

Sills, G. J., and Rogawski, M. A. (2020). Mechanisms of action of currently used antiseizure drugs. Neuropharmacology 168:107966. doi: 10.1016/j.neuropharm.2020.107966
Stafstrom, C. E., and Rho, J. M. (2012). The ketogenic diet as a treatment paradigm for diverse neurological disorders. Front. Pharmacol. 3:59. doi: 10.3389/fphar.2012.00059

Todorova, M. T., Tandon, P., Madore, R. A., Stafstrom, C. E., and Seyfried, T. N. (2000). The ketogenic diet inhibits epileptogenesis in EL mice: a genetic model for idiopathic epilepsy. Epilepsia 41, 933-940. doi: 10.1111/j.1528-1157.2000.tb00275.x

Vergati, M., Krasniqi, E., Monte, G. D., Riondino, S., Vallone, D., Guadagni, F., et al. (2017). Ketogenic diet and other dietary intervention strategies in the treatment of cancer. Curr. Med. Chem. 24, 1170-1185. doi: 10.2174/09298673246661701161 22915

Williams-Karnesky, R. L., Sandau, U. S., Lusardi, T. A., Lytle, N. K., Farrell, J. M., Pritchard, E. M., et al. (2013). Epigenetic changes induced by adenosine augmentation therapy prevent epileptogenesis. J. Clin. Invest. 123, 3552-3563. doi: 10.1172/JCI65636

Zahnow, C. A., Topper, M., Stone, M., Murray-Stewart, T., Li, H., Baylin, S. B., et al. (2016). Inhibitors of DNA methylation, histone deacetylation, and histone demethylation: a perfect combination for cancer therapy. Adv. Cancer Res. 130, 55-111. doi: 10.1016/bs.acr.2016. 01.007

Zubair, A. S., McAlpine, L. S., Gardin, T., Farhadian, S., Kuruvilla, D. E., and Spudich, S. (2020). Neuropathogenesis and neurologic manifestations of the coronaviruses in the age of coronavirus disease 2019: a review. JAMA Neurol. 77, 1018-1027. doi: 10.1001/jamaneurol.20 20.2065

Conflict of Interest: The author declares that the research was conducted in the absence of any commercial or financial relationships that could be construed as a potential conflict of interest.

Copyright (C) 2021 Boison. This is an open-access article distributed under the terms of the Creative Commons Attribution License (CC BY). The use, distribution or reproduction in other forums is permitted, provided the original author(s) and the copyright owner(s) are credited and that the original publication in this journal is cited, in accordance with accepted academic practice. No use, distribution or reproduction is permitted which does not comply with these terms. 